\title{
Frequency and Determinants of Prescribing Antibiotics for Internal Medicine at Donka National Hospital (Guinea)
}

\author{
Salifou Talassone Bangoura ${ }^{1}$, Abdoulaye Toure ${ }^{1,2,3,}$, , Sidikiba Sidibé ${ }^{1}$, Alioune Camara ${ }^{1}$, \\ Djibril Sylla ${ }^{4}$, Alpha-Kabinet Keita ${ }^{2}$, Mamadou Saliou Sow ${ }^{2,5}$, Alimou Camara ${ }^{6}$, \\ Mamadou Saliou Kalifa Diallo ${ }^{2}$, Mohamed Cisse ${ }^{7}$ \\ ${ }^{1}$ Department of Public Health, Gamal Abdel Nasser University of Conakry, Conakry, Guinea \\ ${ }^{2}$ Guinean Research Center and Training in Infectiology (CERFIG), Gamal Abdel Nasser University of Conakry, Conakry, Guinea \\ ${ }^{3}$ National Public Health Institute, Ministere of Health, Conakry, Guinea \\ ${ }^{4}$ Department of Internal Medicine, Donka National Hospital, Conakry, Guinea \\ ${ }^{5}$ Department of Infectious and Tropical Diseases, Donka National Hospital, Conakry, Guinea \\ ${ }^{6}$ Department of Pharmaceutical and Biological Sciences, Gamal Abdel Nasser University of Conakry, Conakry, Guinea \\ ${ }^{7}$ Department of Dermatology and Venerology, Donka National Hospital, Conakry, Guinea
}

\section{Email address:}

abdoulayek2002@yahoo.fr (A. Toure)

${ }^{*}$ Corresponding author

\section{To cite this article:}

Salifou Talassone Bangoura, Abdoulaye Toure, Sidikiba Sidibé, Alioune Camara, Djibril Sylla, Alpha-Kabinet Keita, Mamadou Saliou Sow, Alimou Camara, Mamadou Saliou Kalifa Diallo, Mohamed Cisse. Frequency and Determinants of Prescribing Antibiotics for Internal Medicine at Donka National Hospital (Guinea). Central African Journal of Public Health. Vol. 6, No. 1, 2019, pp. 1-4. doi: $10.11648 /$ j.cajph.20200601.11

Received: November 21, 2019; Accepted: December 17, 2019; Published: January 6, 2020

\begin{abstract}
Background: In hospitals, antibiotics are one of the most prescribed drug classes. The purpose of this study was to describe the extent and factors associated with the prescription of antibiotics in the Internal Medicine Department of Donka National Hospital. Material and Methods: A cross-sectional study was conducted from July 30, 2018 to January 31, 2019 among prescribers and patients hospitalized or seen on an outpatient basis. Data were collected by direct interview and through patient records. A multivariate logistic regression was performed to identify the factors associated with antibiotic prescribing. Results: Out of a total of 400 consultants, $82(21 \%)$ patients received antibiotic therapy. Beta-lactam antibiotics were the most prescribed antibiotic (45\%) and urogenital infections $(24.3 \%)$ were the most common indication. In univariate analysis, the consultation by a trainee physician $[\mathrm{OR}=3.06(95 \% \mathrm{CI}: 1.56-6)]$ was associated with a prescription for antibiotics. Students/students [OR $=1.85$ (95\% CI: 1.01-3.39)], civil servants [OR $=1.82$ (95\% CI: 1.02-3.26)], patients residing in Conakry [OR $=2.06(95 \% \mathrm{CI}: 1.08-3.91)]$, those hospitalized [OR $=3.16(95 \% \mathrm{CI}: 1.55-6.46)]$ and those with a fever [OR $=$ 6.49 (95\% CI: 3.84-10.96)] were more likely to receive an antibiotic. Only fever [OR adjusted = 4.30 (95\% CI: 2.43-7.61)] remained associated with the prescription of antibiotics in multivariate analysis. Conclusion: The results showed that fever was the factor independently associated with the antibiotic prescription. In a country where malaria is the main cause of fever, the results suggest the need for training health care workers in antibiotic prescribing.
\end{abstract}

Keywords: Antibiotic, Frequency, Associated Factors

\section{Introduction}

Antibiotics are natural substances, synthetic or semisynthetic that can inhibit or destroy bacterial agents. [1] In hospitals, they represent one of the most prescribed drug classes. However in 20 to $50 \%$ of cases their prescriptions are inappropriate. [2] In Guinea, a 2012 study reported 63.1\% of non-compliant antibiotic prescriptions for patients with low respiratory infection. [3] Inappropriate prescription of antibiotics is currently a subject of concern and debate in many countries, which needs to be seriously addressed. [4, 5] 
However, this misuse of antibiotics can lead to two major consequences: the emergence of resistant bacterial strains and significant morbidity and mortality. [6] Estimates from a recent study show that drug-resistant infections have caused an estimated 700,000 deaths a year worldwide. [7] This figure is expected to reach 10 million by 2050, with associated costs of up to US \$ 100 billions. [7] To face this threat, it is fundamental to better understand the factors that influence the appropriate prescription. [8] A narrative review of the factors influencing the prescription of antibiotics has revealed the multiple sources of influence affecting a possible prescription. [9] Among which, factors related to the prescribing physician (fear of failure, diagnostic uncertainty or insufficient training), to the patient (history of vulnerable or at risk patients) and to the environment (regulation of prescription and dispensing of drugs and lack of resources for etiological diagnosis). [9] The purpose of this study was to describe the frequency of prescription of antibiotics and the factors associated with this prescription.

\section{Material and Methods}

\subsection{Site and Type of Study}

It was a cross-sectional analytical survey conducted in Conakry from July 30, 2018 to January 31, 2019 at the Internal Medicine Department of Donka National Hospital (Guinea).

This service is one of the two internal medicine departments of Conakry University Hospital. It has a capacity of 18 beds with a staff of 33 peoples and hospitalises on average 481 patients per year.

\subsection{Patients}

The study included patients who were seen outpatient or hospitalized during the study period.

\subsection{Collection of Data}

A standardized survey form was developed and pre-tested before the start of the study. It included the different data for the prescriber (status, year of experience, reasons for antibiotic prescription without bacteriological examination), the sociodemographic data of the patient (sex, age, profession, source), the clinical and biological data of the patient (fever and diagnosis) and therapeutic data (molecule, dosage form, route of administration and duration of treatment).

Data were collected using standardized questionnaires administered to health staff and patients. Clinical and therapeutic data were completed through medical records.

\subsection{Data Analysis}

Data entry was done using the Epi-data software. SPSS version 21 statistical software was used for data analysis. Qualitative data were described by numbers and percentages. Quantitative data were described using the mean, medians and interquartile range. The Khi 2 test was used for the comparison of categorical values. The Student's test was used for the comparison of the averages and that of Wilcoxon for the comparison of the medians. Univariate and multivariate logistic regression was used to identify factors associated with antibiotic prescribing. Only variables with p-value $\leq 20 \%$ in univariate analysis were retained for multivariate logistic regression. A value of $\mathrm{p}<0.05$ was considered statistically significant.

\section{Results}

A total of 400 patients were included in this study. The mean age of the patients surveyed was $43.75 \pm 19.18$ years with a female predominance $(58 \%)$.

Of all patients, 82 received an antibiotic prescription, which corresponds to a frequency of $21.0 \%$ [ $95 \%$ confidence interval (CI): 16.8-25.0].

Prescriptions mainly concerned beta-lactams (45\%), followed by macrolides (16\%), quinolones (13\%), sulfonamides $(13 \%)$ and imidazoles $(12 \%)$. The majority of prescriptions $(86.6 \%)$ contained an antibiotic. Bi-antibiotic therapy was instituted in 10 cases $(12.2 \%)$ of prescription. The compressed form the dosage form was used in 55 cases $(67 \%)$, followed by suspension for injection in form 21 cases $(25.6 \%)$. The other dosage forms used were injectable solutions in 7 cases $(8.5 \%)$ and eggs in 5 cases $(6 \%)$.

The median duration of treatment was 8 days (Interquartile range: 6-10).

In total, 10 different types of diagnosis subject to antibiotic prescription were observed. Urogenital infections (20 cases, $24.3 \%$ ) were the most dominant indication, followed by pneumopathies (16 cases, 19.5\%), ENT diseases (12 cases, $14.6 \%$ ), digestive (12 cases, 14.6\%) and rheumatoid arthritis (8 cases, 9.7\%).

All antibiotic prescriptions were made without bacteriology results. The reasons for prescribing antibiotics without bacteriological examination mentioned by the prescribers were marked by the suspicion of infection (44 cases, $53.5 \%$ ), the presence of leucorrhea (11 cases, 13.4\%), radiographic results. (8 cases, $9.7 \%)$ and tonsillar hypertrophy ( 8 cases, $9.7 \%$ ). The median year of experience of antibiotic prescribers [4: 3-20] was statistically different from that of prescribers who did not prescribe antibiotics [28: 6-36] $(\mathrm{p}<0.05)$.

The results of univariate analysis showed that doctors prescribed trainees 3.06 times more antibiotic that physicians who $[\mathrm{OR}=3.06$ (95\% CI: 1.56 to 6$)]$ (Table 1$)$.

Students / pupils $[\mathrm{OR}=1.85(95 \%$ CI 1.01 to 3.39$)$ ] officials $[\mathrm{OR}=1.82(95 \%$ CI 1.02 to 3.26$)]$, patients residing in Conakry $[\mathrm{OR}=2.06(95 \% \mathrm{CI}: 1.08-3.91)]$, those hospitalized $[\mathrm{OR}=3.16(95 \% \mathrm{CI}: 1.55-6.46)]$ and patients with fever $[\mathrm{OR}=6.49$ (95\% CI: 3.84-10.96)] had the highest odds ratio of receiving an antibiotic (Table 1). In multivariate analysis, fever $[\mathrm{OR}=4.30$ (95\% CI: 2.43-7.61)] was the factor independently associated with antibiotic prescribing (Table 1). 
Table 1. Univariate and multivariate analysis of risk factors for antibiotic prescription in patients in consultation at the Internal Medicine Department of Donka National Hospital from July 30, 2018 to January 31, 2019.

\begin{tabular}{|c|c|c|c|c|c|}
\hline & & Odds ratio p-value & & Adjusted Odds Ratio & p-value \\
\hline \multirow[t]{4}{*}{ Prescibers } & Physician & 1 & & & \\
\hline & Trainee doctor & $3.06(1.56-6)$ & 0.001 & $1.19(0.50-2,80)$ & 0.68 \\
\hline & Year Experience & $0.94(0.92-0.96)$ & 0.0001 & $0.96(0.94-0.98)$ & 0.001 \\
\hline & & Patients & & & \\
\hline \multirow{2}{*}{ Sex } & Man & $1.17(0,72-1,91)$ & 0.52 & - & - \\
\hline & Feminine & 1 & & - & \\
\hline \multirow[t]{2}{*}{ Age (continue) } & & $0.97(0.96-0.99)$ & 0.001 & $0.98(0.97-1)$ & 0.09 \\
\hline & Student & $1.85(1.01-3.39)$ & 0.04 & $0.82(0.42-1.62)$ & 0.57 \\
\hline \multirow[t]{2}{*}{ Profession } & Civil servant & $1.82(1.02-3.26)$ & 0.04 & $1.28(0.62-2.66)$ & 0.49 \\
\hline & Housewife & 1 & & 1 & \\
\hline \multirow{2}{*}{ Provenance } & Conakry & $2.06(1.08-3.91)$ & 0.027 & $0.95(0.44-2.05)$ & 0.91 \\
\hline & Outback & 1 & & 1 & \\
\hline \multirow{2}{*}{ Consultation type } & Hospitalized & $3.16(1.55-6.46)$ & 0.002 & $1,31(0.52-3.31)$ & 0.55 \\
\hline & Ambulatory & 1 & & 1 & \\
\hline Presence of fever & & $6.49(3.84-10.96)$ & 0.0001 & $4.30(2.43-7.61)$ & 0.0001 \\
\hline
\end{tabular}

\section{Discussion}

The main objective of this study was to describe the frequency of prescription of antibiotics and the factors associated with this prescription. The results of this study showed that $21 \%$ of inpatients or outpatients in the internal medicine department received an antibiotic prescription. All these antibiotic prescriptions were empirical, which is contrary to the guidelines and recommendations. [10] This frequency of antibiotic therapy in our study was higher than that reported in Dakar by Déguénonvo LF et al. (12.5\%), [11] but less than the $39.8 \%$ reported in Mali in the pediatric ward. [12] This increased prescription of antibiotics without biological results is due to the fact that doctors, especially young doctors, tend to believe that any case of fever evokes either malaria or a bacterial infection. This could imply a weak adherence to international recommendations. It could also be explained by the fact that services have not appointed a prescribing antibiotics doctor. In addition, the low capacity of laboratories to carry out bacteriological examinations with antibiogram, in particular, blood cultures could explain the low access of patients to laboratory tests.

The most prescribed families of antibiotics were betalactams, macrolides, quinolones and sulfonamides. These results are comparable to those reported by Dial NM et al. where the prescribing frequencies were $55.43 \%$ for betalactams, $16.5 \%$ for macrolides and $13.9 \%$ for imidazoles, [13] but in the Indian medicine service, the most commonly prescribed antibiotics were third-generation cephalosporins, amoxicillin / clavulanic acid and quinolones. [14]

The bioavailability and / or broad spectrum of beta-lactams may explain their predominance since they were only probabilistic prescriptions.

Bi-antibiotic therapy was instituted in 10 cases of prescription. These results are similar to those of a study in Bangladesh where only one antibiotic was prescribed in $74.56 \%$ of cases, while two or more antibiotics were prescribed in $25.44 \%$ of cases. [15]

Our study found that compressed antibiotic prescriptions accounted for a high proportion of all antibiotic prescriptions. This is consistent with WHO data from, that recommends prioritizing oral antibiotics when using antibiotics and using injectable antibiotics only when necessary. [16] However different from that of Alemayehu et al. in the Oromia region of Ethiopia, who found that most antibiotics prescribed were administered intravenously (38.2\%), followed by Per-os (34.1\%). [17]

A large proportion of antibiotic prescriptions were intended for urogenital infections. Elbouti et al. reported a similar proportion (24.8\%). [8] In Benin, Affolabi et al. also reported in their study that uro-genital infections $(28.6 \%)$ were the leading causes of antibiotic prescription in adolescents and adults. [18]

Our results showed that patients with fever were more likely to receive antibiotic prescriptions. This is the same in a study conducted in primary and secondary health care facilities in Uttar Pradesh, India, where $85 \%$ of patients with fever received antibiotics. [19] Fever is a common symptom of many diseases such as malaria, viral fever, bacterial infections etc. As a result, doctors may have prescribed antibiotics as a probabilistic treatment to treat a bacterial infection if necessary.

This study has some limitations that need to be clarified. It is, among other things, its transversal nature. The methodology used did not allow us to properly describe the duration of exposure of patients to antibiotics, nor to document the changes in prescription. It also failed to analyze the impact of poor prescription on patient survival. It is however, to our knowledge, one of the few studies that have evaluated the extent of prescription of antibiotics at Conakry University Hospital.

\section{Conclusion}

In sum, this study has shown that one out of five patients received in consultation or hospitalized in the internal medicine department of the National Hospital has received an antibiotic prescription. The factor independently associated with the prescription of these antibiotics was the occurrence 
of fever in the patient. The prescriptions were made mainly by young people who have less work experience and the main family of antibiotics prescribed was beta-lactams. These results suggest the need for better education and training of young people in the various departments to avoid inappropriate prescription antibiotics.

\section{Conflict of Interest}

The authors declare that they have no conflict of interest.

\section{Author's Statement}

The design of the study was done by Abdoulaye Touré and Salifou Talassone Bangoura. Data acquisition, statistical analysis and manuscript writing were carried out by Salifou Talassone Bangoura under the supervision of Abdoulaye Touré and Mamadou Saliou Kalifa Diallo. The critical review of the manuscript was done by Abdoulaye Toure, Sidikiba Sidibé, Alioune Camara, Djibril Sylla, Alpha-Kabinet Keita, Mamadou Saliou Sow, Alimou Camara, Mohamed Cisse. All authors have read and approved the final manuscript.

\section{Acknowledgements}

I would like to thank all the authors for their collaboration, their precious help, their proofreading and corrections. I would also like to thank the entire team at the Infectious Diseases Research and Training Centre in Guinea and the Internal Medicine Department of Donka National Hospital for their support.

\section{References}

[1] Bevilacqua S, Boschetti E, Lozniewski A and al. Policy proper use of antibiotics: the example of Nancy University Hospital. Med Mal Infect 2007; 37: 32-33.

[2] Roger PM, Labate C, Brofferio $\mathrm{P}$ and al. Prospective evaluation of antibiotic associations by the reference infectious disease specialist of a hospital center. Med Mal Infect 2008; 38: 159.

[3] Sow MS, Camara A, Fortes Déguénonvo L and al. Evaluation of the prescription of antibiotics in the lower respiratory tract infections in adults at the University Hospital of Conakry in Guinea. Rev. CAMES-Série A 2012; 13: 239-243.

[4] Huibers L, Moth G, Christensen MB and Vedsted P. Antibiotic prescribing patterns in out-of-hours primary care: A population-based descriptive study. Scandinavian Journal of Primary Health Care 2014; 32: 200-207.

[5] Cordoba G, Siersma V, Lopez-Valcarcel B and al. Prescribing style and variation in antibiotic prescriptions for sore throat: cross-sectional study across six countries. BMC Family Practice 2015; 16: 1-8.
[6] Elbouti A, Rafai M, Chouaib $\mathrm{N}$ and al. Evaluation of antibiotic prescriptions in the Emergency Department of the Military Hospital of Instruction Mohammed V (HMIMV). Pan Afr Med J 2016; 25: 162.

[7] Anna Machowska and Cecilia Stålsby Lundborg. Drivers of Irrational Use of Antibiotics in Europe. Int. J. Environ. Res. Public Health 2019; 16: 27.

[8] Spoorenberg V, Geerlings SE, Geskus RB and al. Appropriate antibiotic use for patients with complicated urinary tract infections in 38 Dutch Hospital Departments: a retrospective study of variation and determinants. BMC Infectious Diseases 2015; 15: 505.

[9] McKay R, Mah A, Law MR and al. Systematic Review of Factors Associated with Antibiotic Prescribing for Respiratory Tract Infections. Antimicrob Agents Chemother 2016; 60: 184106.

[10] National Center for Disease Control in India. National Treatment Guidelines of India for the Use of Antimicrobials in Infectious Diseases. Version 1.0. New Delhi, 2016: 1-64.

[11] Déguénonvo LF, Diallo MOS, Badiane NMD and al. Evaluation of the quality of the antibiotic prescription at the Fann CHNU Emergency Reception Service in Dakar. Méd. et maladies infect 2013; 43: 78.

[12] Coulibaly Y, Konate A, Kone D and Bougoudogo F. Study of the prescription of antibiotics in Malian hospital. Rev Malienne d'Infectiologie Microbiol 2014; 3: 2-8.

[13] Dial NM, Ismail YZ, Lakhe NA and al. Evaluation of the Quality of Prescription of Antibiotics with Curative Intent in a Specialized Department in Dakar, Senegal. Arch Infect Dis Ther 2018; 2: 2577-8455.

[14] Alvarez-Uria G, Zachariah S and Thomas D. High prescription of antimicrobials in a rural district hospital in India. Pharm Pract (Grenade) 2014; 12: 384.

[15] Biswas M, Roy DN, Tajmim A and al. Prescription antibiotics for outpatients in Bangladesh: a cross-sectional health survey conducted in three cities. Annals of Clinical Microbiology and Antimicrobials 2014; 13: 15.

[16] WHO Policy Perspectives on Medicines. Promote the rational use of medicines: essential components. Geneva: World Health Organization; 2002; 6.

[17] Alemayehu GA, Gonfa R and Tadesse T. Evaluation of Antibiotic Use in Medical Ward of Fitche District Hospital, North Showa Zone, Oromia Region, Ethiopia. Adv Pharmacoepidemiol Drug Saf; 2017; 6: 2167-1052.

[18] Affolabi D, Alidjinou KE, Dossou-Gbété L and al. Assessment of antibiotics prescribing for outpatients in Cotonou, Benin. Bull Soc Pathol Exot 2009; 102: 110-112.

[19] Kumar R, Indira K, Rizvi A and al. Antibiotic prescribing practices in primary and secondary health care facilities in Uttar Pradesh, India. J Clin Pharm Ther 2008; 33: 625-3. 